\title{
A Global Optimization Approach Applied to Structural Dynamic Updating
}

\author{
Marco Dourado ${ }^{1}$, José Meireles ${ }^{2}$, Ana Maria A. C. Rocha ${ }^{3}$ \\ ${ }^{1} \mathrm{PhD}$ Student, Department of Mechanical Engineering, Centre for Mechanical and Materials \\ Technologies (CT2M), University of Minho, Portugal \\ mdourado@dem. uminho.pt \\ ${ }^{3}$ Department of Mechanical Engineering, Centre for Mechanical and Materials Technologies \\ (CT2M), University of Minho, Portugal \\ meireles@dem.uminho.pt \\ ${ }^{3}$ Department of Production and Systems, Algoritmi Research Centre, University of Minho, \\ Portugal \\ arochaddps.uminho.pt
}

\begin{abstract}
In this paper, the application of stochastic global optimization techniques, in particular the GlobalSearch and MultiStart solvers from MatLab ${ }^{\circledR}$, to improve the updating of a structural dynamic model, are presented. For comparative purposes, the efficiency of these global methods relatively to the local search method previously used in a Finite Element Model Updating program is evaluated. The obtained solutions showed that the GlobalSearch and MultiStart solvers are able to achieve a better solution than the local solver previously used, in the updating of a structural dynamic model. The results show also that the GlobalSearch solver is more efficient than the MultiStart, since requires less computational effort to obtain the global solution.
\end{abstract}

Keywords: Finite Element Model Updating, Global Optimization, Structural Dynamic

\section{Introduction}

Optimization problems can go from simple linear functions with few variables, until the most complex problems of non-linear functions, with many variables, with constraints on the variables and many optimal local solutions [1].

Depending on the problem under study, local or global optimization methods can be used to find the maximum or minimum of a function. The selection of a method for a particular application depends on the characteristics of the problem and what is desired, such as type of design variables, whether or not all local minima are desired, and availability of gradients of the functions. Many engineering optimization problems are multimodal and require the application of global search methodologies, in order to avoid the optimizer to be trapped in the first minimum or maximum local found. The global search methodologies allow the optimizer to evolve into other areas of the feasible region, being possible to obtain more and best solutions. 
There are two major classes of methods depending on whether or not they incorporate any stochastic elements to solve the global optimization problem: deterministic and stochastic methods.

Deterministic methods provide a theoretical guarantee of locating the global minimum. Stochastic methods only give guarantee in a probabilistic sense that the global minimum point will be found. On the other hand, stochastic methods are usually faster in locating a global optimum than deterministic ones. In most global optimization algorithms (both deterministic and stochastic) it is possible to identify two phases: a global phase and a local phase. The exhaustive exploration of the search space is delegated to the global phase, where the function is evaluated at a number of randomly sampled points. In the local phase, the sample points are manipulated, by means of local searches, to yield a candidate global minimum [2]. For an introduction to deterministic and stochastic methods in global optimization, see e.g. Horst and Tuy [3] and Törn and Zilinskas [4], respectively.

There are some examples of application of deterministic methods in structural engineering area, such as the work of Stolpe [5], that presents a branch-and-bound algorithm for global optimization of the minimum weight stress-constrained truss topology problem extended with displacement bounds and local buckling constraints. Later, using the same algorithm, Achtziger and Stolpe [6] developed a study to determine the optimal variables of a truss structure. However, Lin and Chen [7] emphasize that deterministic methods are based on assumptions of objective and constraint functions, and therefore the deterministic methods cannot be applied to general structural problems with satisfactory efficiency.

Thus, the stochastic methods became relevant to solve the most global optimization problems, since they adapt better to real problems or black-box formulations, and they prove to be very useful for applications in the field of structural engineering optimization problems, as for example, presented by Lucor [8]. They were inspired on natural environmental, biological, physical and chemical processes, composed by populations of individuals or elements that interact between them, and with their environment. The algorithms based on these natural phenomena are called Swarm Intelligence [9]. Those social behaviors have been crucial for the development of the random search methods and multi start methods. Some examples of application of these two subclasses of stochastic methods are the work of Lin and Chen [7] to study multistage optimization algorithms for simultaneously seeking multiple optimal solutions in a structural problem and Eriksson and Arora [10] to study the efficiency of three global stochastic optimization algorithms, with continuous variables, in the optimization of the ride comfort of a city bus. Within the stochastic methods are also the sub-class of evolutionary methods, such as Genetic Algorithms (GA), and Simulated Annealing (SA), used by Sonmez [11] to obtain multi optimal shapes for two-dimensional structures subject to quasi-static loads and restraints, and Venanzi e Materazzi [12] to optimize wind-excited structures. The hybridization of a genetic algorithm and a nonsmooth proximal bundle method is used in Auvinen et al. [13] to minimize the weight of a forest machine, and Keller [14] applied evolutionary algorithms to a case study of an air-plane's side rudder. 
This paper intends to show the application of global stochastic optimization methods, in the structural engineering field, namely in the optimization of structural dynamic models with resort to methods of improving finite element models, usually denoted by Finite Element Model Updating. These improvements can be conducted under two types of approach:

1. in the updating of simplified numerical models, representative of detailed physical models which present high computation time. The simplified model is submitted to updating by a Finite Element Model Updating methodology until obtain dynamic behavior similar to the physical model, also denominated as reference model [15]. Thus, it is possible to obtain a light computationally model and at the same time representative of the physical model. It is important to refer that, in these cases, the main interest is in the correlation of dynamic behavior, independently of the parameters values optimized;

2. in the structural modification to the optimization of the models. Detailed numerical models of physical models are built and submitted to optimization to: improve the dynamic behavior and/or achieve a model with similar behavior but with geometrical and/or physical parameters more advantageous from the design point of view [16].

The optimization methodologies help to fit on the control of updating process, nevertheless still constitute a developing task. Important works in the Finite Element Model Updating area, using global stochastic optimization methods, could be found in Levin and Lieven [17], that compares various implementations of two algorithms, the GA and SA, to find the global minimum, amongst many local minima, of an objective function that describes the finite element model updating of a flat plate wing structure in the frequency domain. Teughels et al. [18] use the Coupled Local Minimizers (CLM) method in the Finite Element Model Updating program for the damage identification of a reinforced concrete beam. The method combines the fast convergence of the local gradient-based algorithms with the global approach of GA, resulting in an efficient global optimization algorithm, able to find the global minimum of the objective function. The same method was used by Bakir et al. [19] to update the finite element model of a reinforced concrete frame, using 24 design variables. The authors compare the CLM method with different optimization local search methods, such as the Gauss-Newton method, Levenberg-Marquardt algorithm and Sequential Quadratic Programming (SQP) algorithm, and prove that the global method gave better results. Ameri et al. [20] used the Globalized Bounded Nelder-Mead method to find the optimal fiber orientation of laminated cylindrical panels based on natural frequencies by maximization of fundamental natural frequency. The obtained results show good accuracy and cost optimization when compared with results of GA.

Following the same principles of the cited authors, and in order to improve the efficiency of a Finite Element Model Updating program, two global stochastic optimization techniques, the GlobalSearch and MultiStart commands available in Matlab ${ }^{\circledR}$, are used and compared with each other when applied to the updating process of a structural model. The aim is to compare the obtained solutions with the local solutions previously obtained in the Finite Element Model Updating program, developed by 
Meireles [21,22]. This Finite Element Model Updating program, has implemented in its optimizer a local search method that uses the SQP algorithm performed through the fmincon command from Matlab ${ }^{\circledR}$ to find the optimal global value. However, this implemented local search strategy, have difficulties to reach the global optimum, since it was developed to find local solutions.

The organization of this paper is as follows. Section 2 presents the mathematical formulation of the problem. Section 3 describes the optimization process and the models description used in the optimization process are presented in Section 4. Section 5 shows the computational experiments done with the local and the global solvers as well as a discussion of the obtained solutions. This paper is concluded in Section 6.

\section{Problem Formulation}

The optimization problem consists in the minimization of an objective function, related with the frequencies and respective mode shapes correlation between the reference model and the numerical model, defined as

$$
\begin{array}{cc}
\min & f(x) \\
\text { s.t. } & x_{L B} \leq x \leq x_{U B}
\end{array}
$$

where $x$ is the vector with the updated parameters for the numerical model, and $x_{L B}$, and $x_{U B}$ are the lower and upper bounds on the variables, respectively.

The objective function of the optimization problem is defined by the sum of three specific functions, as

$$
f(x)=f_{\varphi C}(x)+f_{\varphi U}(x)+f_{\lambda}(x)
$$

where $f_{\varphi C}(x)$ represents the quantification of the difference between numerical and reference correlated mode pairs, $f_{\varphi U}(x)$ represents the quantification of the difference between numerical and reference uncorrelated mode pairs and $f_{\lambda}(x)$ represents the quantification of the difference between numerical and reference frequencies.

Function $\mathrm{f}_{\varphi \mathrm{C}}(\mathrm{x})$ is given by

$$
f_{\varphi C}(x)=-\frac{\sum_{i=1}^{N_{C}} M A C_{i i}(x)}{\sum_{i=1}^{N_{C}} M A C_{i i}\left(x^{0}\right)}
$$

where $N_{C}$ is the number of correlated mode pair values of the diagonal MAC matrix and the vector $x^{0}$ contains the initial updating parameters. The $M A C$ matrix is defined by

$$
M A C_{i j}(x)=\frac{\left(\left(\varphi_{i}^{\text {Ref }}\right)^{T} \varphi_{j}^{\text {Num }}\right)^{2}}{\left(\left(\varphi_{i}^{\text {Ref }}\right)^{T} \varphi_{i}^{\text {Ref }}\right)\left(\left(\varphi_{j}^{\text {Num }}\right)^{T} \varphi_{j}^{\text {Num }}\right)}
$$

where, $\varphi_{i}^{\text {Ref }}$ is the $i$ th reference mode shape and $\varphi_{j}^{\text {Num }}$ is the $j$ th numerical mode shape [22]. 
Function $f_{\varphi U}(x)$ is given by

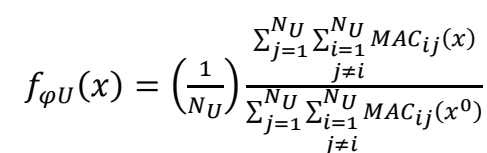

where $N_{U}$ is the number of uncorrelated mode pairs values, outside of the diagonal $M A C$ matrix.

Function $f_{\lambda}(x)$ represents the quantification of the difference between numerical and reference frequencies, given by

$$
f_{\lambda}(x)=-\frac{\sum_{i=1}^{N_{\lambda}}\left(\omega_{i}^{R e f}-\omega_{i}^{N u m}(x)\right)^{2}}{\sum_{i=1}^{N_{\lambda}}\left(\omega_{i}^{R e f}-\omega_{i}^{N u m}\left(x^{0}\right)\right)^{2}}
$$

where $N_{\lambda}$ is the number of eigenvalues $\lambda$ corresponding to the correlated mode pairs, $\omega^{R e f}$ is the reference frequency and $\omega^{N u m}$ is the numerical frequency, respectively defined by $\omega^{R e f}=\sqrt{\lambda^{R e f} / 2 \pi}$ and $\omega^{\text {Num }}=\sqrt{\lambda^{N u m} / 2 \pi}$. The quadratic term in (6) is used to accelerate the convergence and to obtain only positive differences between the frequencies of the two models. Numerical mode shapes $\varphi^{\text {Num }}$ and numerical eigenvalues $\lambda^{\text {Num }}$ are function of these updating parameters. The relationship between them can be written as

$$
\left(\varphi^{N u m}, \lambda^{N u m}\right)=f\left(x_{1}, x_{2}, x_{3}, \ldots, x_{p}\right)
$$

where $p$ is the number of updating parameters. The updated physical parameters $x$, that represent the best improvement of the numerical model, are obtained when the objective function (2) is minimized.

\section{Optimization Process}

The optimization process uses the interaction between Matlab ${ }^{\circledR}$ and Ansys ${ }^{\circledR}$ to improve the dynamic characteristics of the numerical model calculating the objective function value and finding the optimal value of the physical parameters.

The flowchart of the interaction algorithm between optimization method in Matlab ${ }^{\circledR}$ and Ansys ${ }^{\circledR}$ is represented in Fig.1.

The first step of the structural optimization process is to idealize the desired behavior of the dynamic model to develop, or collect experimental data of a physical model considered as the reference model. The next step is associated with the construction of a numerical model in the finite element program ANSYS ${ }^{\circledR}$, that should describe the idealized dynamic model from which its dynamic characteristics are obtained. These dynamic characteristics are transferred to the optimizer of the Finite Element Model Updating program developed in MatLab ${ }^{\circledR}$, in order to optimize the dynamic behavior of the numerical model when compared to the reference model. It is considered that 
the type of structure, in that this methodology is applicable, is sufficiently rigid that the damping can be neglected.

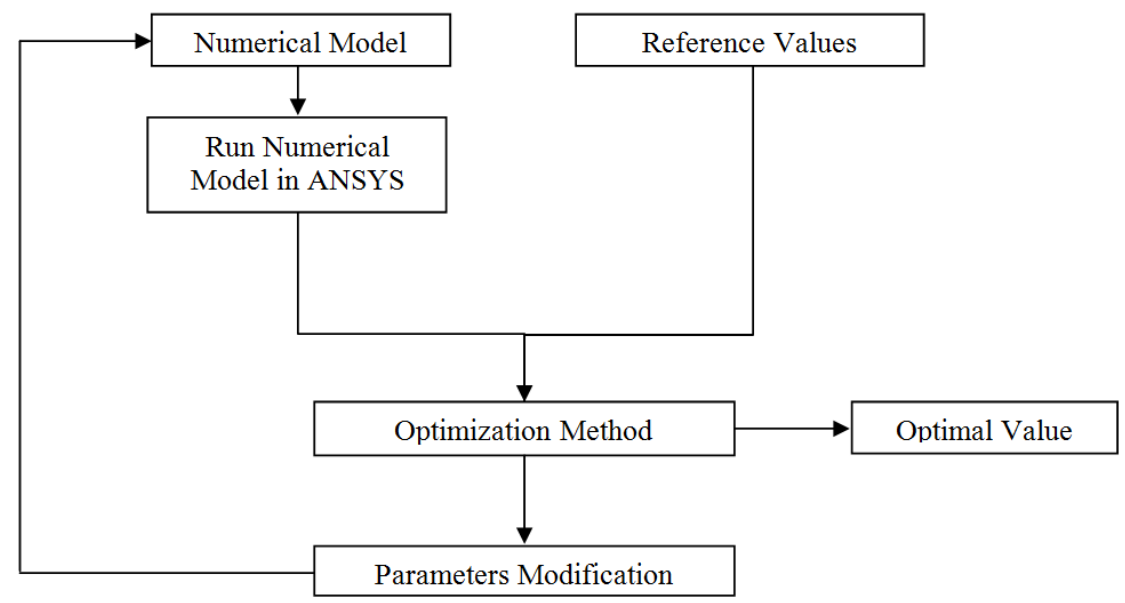

Fig. 1. Interaction flowchart between Matlab ${ }^{\circledR}$ and Ansys ${ }^{\circledR}$

In this study, the Finite Element Model Updating program, implemented in MatLab $^{\circledR}$, uses a global solver, provided by the Global Optimization Toolbox [23] that searches for the optimal global value of the objective function (1). Two global solvers are used in the optimization process, performed by GlobalSearch and MultiStart commands, in order to test its efficiency and effectiveness in the updating process of a structural model. A prior version of the Finite Element Model Updating program uses the local solver, provided by the command fmincon from Matlab [21,22]. Following, the referred commands are briefly introduced.

The fmincon command aims to find a minimum of a constrained problem of multiple variables. Given an initial starting point, this solver can work with four algorithms type: active-set, interior-point, SQP and trust-region-reflective. As described in [23], the active-set and SQP algorithms work of similar way. In these algorithms, a Quadratic Programming subproblem is solved, where, at each iteration, the BFGS (Broyden-Fletcher-Goldfarb-Shanno) formulae is used to estimate the Hessian of the Lagrangian function. The interior-point algorithm is an approach to solve a sequence of approximate minimization problems. The trust-region-reflective algorithm is a subspace trust-region method and is based on the interior-reflective Newton method. Here, each iteration involves the approximate solution of a large linear system using the method of Preconditioned Conjugate Gradients. A work with application of this method can be found in Voormeren and Rixen [24].

GlobalSearch and MultiStart implement stochastic search methods and are similar when finding global or multiple solutions. Both algorithms use multiple start points to sample multiple basins of attraction and start a local solver, such as fmincon, from a variety of starting points and store local and global solutions found during the search process. Generally the starting points are random. 
The GlobalSearch solver performs in two phases: a local phase and a global phase. In the local phase, the sampled points, randomly obtained, are manipulated by a local search to find candidates for local minimum. In the global phase the local minimum with best objective function value is used as an approximation to the global optimum. The solver uses a scatter search strategy in order to generate the trial points. Then, it analyzes the start points and rejects all of those that are unlikely to improve the best local minimum found so far.

The MultiStart solver uses uniformly distributed start points within bounds, or user-supplied start points. Then, it runs the local solver at all start points, or, optionally, all start points that are feasible with respect to bounds or inequality constraints.

\section{Models Description}

In this section the models description is presented, where a numerical model will be optimized taking into account a reference model from which are extracted the reference values of mode shapes and respective natural frequencies.

\subsection{Reference Model}

The reference model is a steel sheet with dimensions $200 \times 300 \times 10 \mathrm{~mm}^{3}$, represented by width $(w)$, height $(h)$ and thickness $(t)$, as shown in Fig.2.
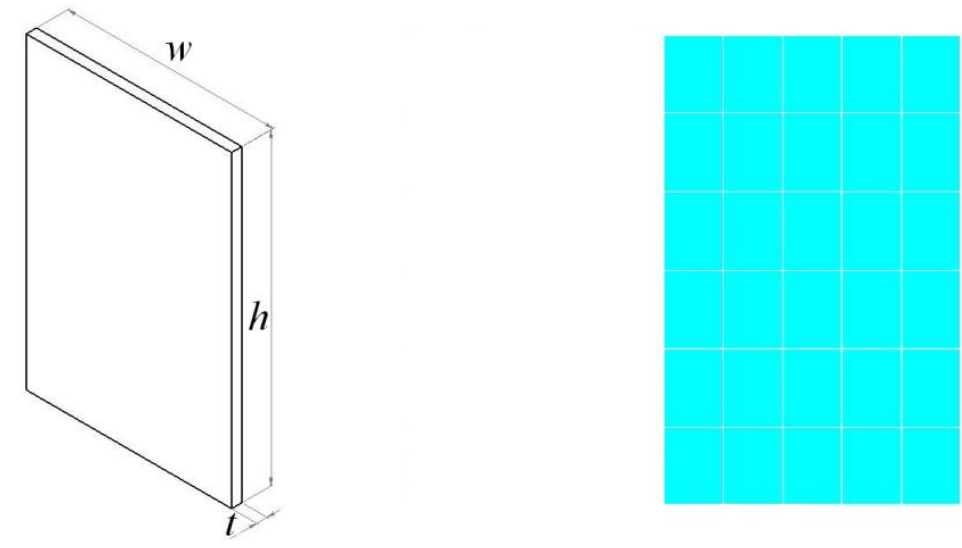

Fig. 2. Reference model

This model is built in ANSYS ${ }^{\circledR}$ with shell elements (SHELL63), and is submitted to modal analysis for extraction of mode shapes measured in 24 points and respective natural frequencies. The mechanical properties of the steel sheet are presented in Table 1 . 
Table 1. Mechanical properties of the reference model

\begin{tabular}{cccc}
\hline Property & Symbol & Units & Value \\
\hline Young's Module & $E_{x}$ & $\mathrm{~Pa}$ & $2.1 \times 10^{11}$ \\
Young's Module & $E_{y}=E_{z}$ & $\mathrm{~Pa}$ & $2.2 \times 10^{11}$ \\
Poisson's Ratio & $v_{x y}=v_{y z}=v_{z x}$ & - & 0.27 \\
Density & $\rho$ & $\mathrm{kg} / \mathrm{m}^{3}$ & 7847 \\
\hline
\end{tabular}

\subsection{Numerical Model}

The numerical model to be optimized has a set of 240 areas of variable geometry, as shown in Fig.3. The areas are created from points, some with variable coordinates, enabling the change of all areas of the model. The points with variable coordinates are function of the geometrical parameters: width $\left(w_{a}\right)$ and height $\left(h_{b}\right)$. The coordinates of the points chosen for reading the mode shapes are kept constant in order to coincide with the readings of reference.

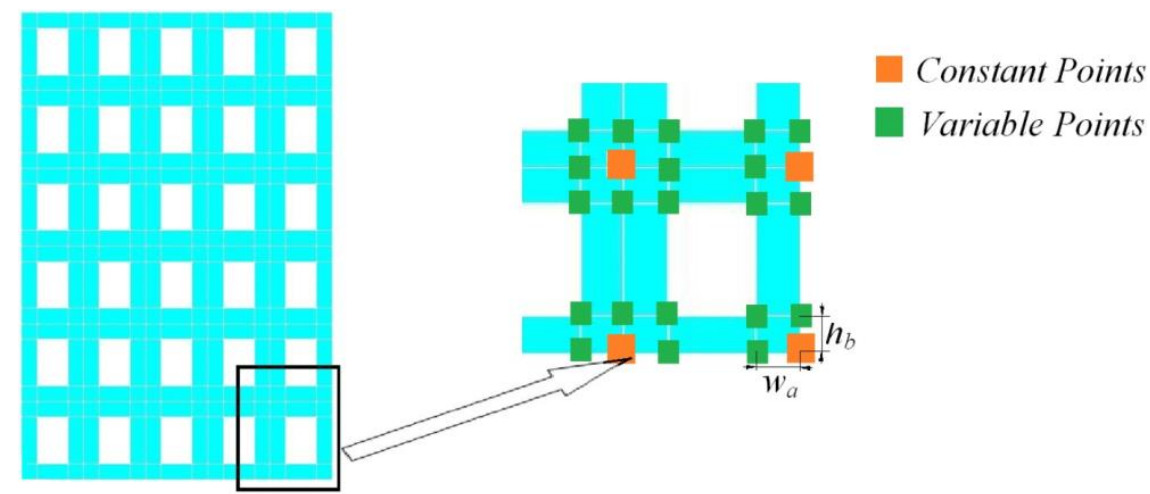

Fig. 3. Initial numerical model

The numerical model built in ANSYS ${ }^{\circledR}$ with shell elements (SHELL63), has the mechanical properties presented in Table 1 . The width $(w)$, height $(h)$ and thickness $(t)$ dimensions are equal to the reference model, represented in Fig.2. The numerical model will be submitted to modifications of geometric parameters, such as thickness $(t)$, width $\left(w_{a}\right)$ and height $\left(h_{b}\right)$, through the optimization process in the Finite Element Model Updating program. The initial values of the parameters and their lower and upper bounds are indicated in Table 2.

Table 2. Parameters vector of the numerical model

\begin{tabular}{cccccc}
\hline Property & Variable & Units & $\begin{array}{c}\text { Initial } \\
\text { Value }\end{array}$ & $\begin{array}{c}\text { Lower } \\
\text { bound }\end{array}$ & $\begin{array}{r}\text { Upper } \\
\text { bound }\end{array}$ \\
\hline Thickness & $t$ & $\mathrm{~mm}$ & 10 & 1 & 20 \\
Width a & $w_{a}$ & $\mathrm{~mm}$ & 10 & 10 & 19 \\
Height b & $h_{b}$ & $\mathrm{~mm}$ & 10 & 10 & 24 \\
\hline
\end{tabular}


It is expected that the optimal value of width $\left(w_{a}\right)$ and height $\left(h_{b}\right)$ variables, have a clear tendency to converge to the upper bound, in order to fill the empty spaces of the steel sheet.

\section{Computational Experiments}

In this section the numerical model is optimized and the computational results are presented. First, the solutions obtained with the local solver fmincon are showed, and then the ones with each of the global solver, GlobalSearch and MultiStart.

The local solver fmincon is performed on the supplied initial point $x^{0}$ with the $a c$ tive-set, interior-point, SQP and trust-region-reflective algorithms. The GlobalSearch solver is performed with 100 and 400 trial points, where the number of points analyzed in stage one is 100 and 400, respectively. So, the GlobalSearch applies the fmincon solver, first, in the supplied initial point $x^{0}$ and, second, in the starting points defined in the option NumStageOnePoints, making only an initial assessment of the score function of each one. Finally the GlobalSearch applies the fmincon solver in the point with best score. Therefore, the GlobalSearch solver makes complete evaluation in only two points, in the supplied initial point $x^{0}$ and in the best starting point among the trial points of stage one. The MultiStart solver is performed with 10 and 20 trial points, and fmincon solver is executed in all of them, and the evaluation is complete for all of them. All the others optional parameter values of the optimization solvers have the default values.

\subsection{Local Solver Results}

For the local solver fmincon analysis, the search is only performed on the starting point $x^{0}$ and theoptimization results are presented in Table 3.

Table 3.Optimization results for fmincon solver

\begin{tabular}{|c|c|c|c|c|c|}
\hline \multicolumn{2}{|c|}{ Output } & active-set & interior-point & SQP & trust-region \\
\hline \multirow{2}{*}{\multicolumn{2}{|c|}{$\begin{array}{l}\text { Nr. function evaluations } \\
\text { Optimization time }[\mathrm{h}]\end{array}$}} & 162 & 138 & 239 & 162 \\
\hline & & $\sim 1.700$ & $\sim 1.500$ & $\sim 2.500$ & $\sim 1.700$ \\
\hline \multirow{3}{*}{$\mathrm{x}$ local $[\mathrm{mm}]$} & $t$ & 10.160 & 10.158 & 10.142 & 10.160 \\
\hline & $w_{a}$ & 14.582 & 14.677 & 15.318 & 14.582 \\
\hline & $h_{b}$ & 14.881 & 14.843 & 14.528 & 14.881 \\
\hline \multicolumn{2}{|c|}{ Optimal local value $f(x)$} & 4.402 & 4.401 & 4.393 & 4.402 \\
\hline
\end{tabular}

The best optimal value found, 4.393, is achieved by SQP algorithm. With the other algorithms the optimal value of $f(x)$ is very similar between them. The solver requires 239 function evaluations and 2.5 hours to achieve the best optimal local value of the objective function. 


\subsection{GlobalSearch Solver Results}

In the GlobalSearch solver analysis, there are, in general, improvements relatively to the local solver solution. In the first experiment, with 100 trial points, where optimization results are presented in Table 4, the SQP algorithm obtains the optimal value of 4.163 , which means an improvement of $5.236 \%$ when compared with the optimal solution obtained with the local search method (4.393). With the other algorithms, only the interior-point achieves a slight improvement of $0.068 \%$ when compared with the value obtained with the same algorithm (4.401). With the active-set and trustregion-reflective algorithms do not found any improvement there. The Globalsearch solver with the SQP algorithm requires 532 function evaluations and 5.5 hours to achieve the best optimal global value, requiring approximately twice more (120\%) of optimization time and function evaluations than with the local solver.

Table 4. Optimization results for GlobalSearch with 100 trial points

\begin{tabular}{|c|c|c|c|c|c|}
\hline \multicolumn{2}{|c|}{ Output } & active-set & interior-point & SQP & trust-region \\
\hline \multicolumn{2}{|c|}{ Nr. function evaluations } & 526 & 505 & 532 & 526 \\
\hline \multicolumn{2}{|c|}{ Optimization time $[\mathrm{h}]$} & $\sim 5.500$ & $\sim 5.200$ & $\sim 5.500$ & $\sim 5.500$ \\
\hline \multirow{3}{*}{$x$ global $[\mathrm{mm}]$} & $t$ & 10.160 & 10.152 & 9.990 & 10.160 \\
\hline & $w_{a}$ & 14.582 & 14.878 & 19.000 & 14.582 \\
\hline & $h_{b}$ & 14.881 & 14.762 & 10.000 & 14.881 \\
\hline \multicolumn{2}{|c|}{ Optimal global value $f(x)$} & 4.402 & 4.398 & 4.163 & 4.402 \\
\hline
\end{tabular}

When using 400 trial points (see Table 5), the results are about the same although a large computational time (more than double).

Table 5. Optimization results for GlobalSearch with 400 trial points

\begin{tabular}{|c|c|c|c|c|c|}
\hline \multicolumn{2}{|c|}{ Output } & active-set & interior-point & SQP & trust-region- \\
\hline \multicolumn{2}{|c|}{ Nr. function evaluations } & 1149 & 1224 & 1168 & 1215 \\
\hline \multicolumn{2}{|c|}{ Optimization time $[\mathrm{h}]$} & $\sim 12.500$ & $\sim 13.300$ & $\sim 12.800$ & $\sim 13.200$ \\
\hline \multirow{3}{*}{$x$ global $[\mathrm{mm}]$} & $t$ & 10.145 & 10.135 & 9.990 & 10.138 \\
\hline & $w_{a}$ & 15.137 & 15.440 & 19.000 & 15.389 \\
\hline & $h_{b}$ & 14.645 & 14.517 & 10.000 & 14.511 \\
\hline \multicolumn{2}{|c|}{ Optimal global value $f(x)$} & 4.394 & 4.391 & 4.163 & 4.392 \\
\hline
\end{tabular}

The active-set algorithm achieves a little improvement of $0.182 \%$ compared with the value obtained with the local solver (4.402). The interior-point and trust-regionreflective algorithms achieve a slight improvement of $0.227 \%$ compared with the value obtained with the local solver (4.401 and 4.402, respectively). The optimal global value obtained with the SQP algorithm remains in 4.163. The solver requires 1168 function evaluations and 12.8 hours to achieve the best optimal global value of the objective function, needing approximately five times more (412\%) of optimization time and function evaluations than with the local solver. 


\subsection{MultiStart Solver Results}

In the MultiStart solver analysis, when the search is performed with 10 trial points (see results in Table 6), the four algorithms have a favorable evolution, when compared to local solver results. The active-set and trust-region-reflective algorithms achieve a slight improvement of $0.250 \%$ compared with the value obtained with the local solver (4.402). The interior-point algorithm achieves a little improvement of $0.182 \%$ compared with the value obtained with the local solver (4.401). The SQP algorithm achieves an improvement of 5.236\% compared with the value obtained with the local solver (4.393) and achieve the best optimal global value of function $f(x)$ (4.163). The solver requires 1735 function evaluations and 19 hours to achieve the best optimal global value of the objective function, needing approximately 7.6 times more (660\%) of optimization time and function evaluations than with the local solver.

Table 6. Optimization results with MultiStart for 10 trial points

\begin{tabular}{|c|c|c|c|c|c|}
\hline \multicolumn{2}{|c|}{ Output } & active-set & interior-point & SQP & trust-region- \\
\hline \multirow{2}{*}{\multicolumn{2}{|c|}{$\begin{array}{l}\text { Nr. function evaluations } \\
\text { Optimization time }[\mathrm{h}]\end{array}$}} & 1553 & 1628 & 1735 & 1665 \\
\hline & & $\sim 17.000$ & $\sim 17.800$ & $\sim 19.000$ & $\sim 18.200$ \\
\hline \multirow{3}{*}{$x$ global $[\mathrm{mm}]$} & $t$ & 10.135 & 10.140 & 9.990 & 10.137 \\
\hline & $w_{a}$ & 15.433 & 15.279 & 19.000 & 15.400 \\
\hline & $h_{b}$ & 14.519 & 14.596 & 10.000 & 14.522 \\
\hline \multicolumn{2}{|c|}{ Optimal global value $f(x)$} & 4.391 & 4.393 & 4.163 & 4.391 \\
\hline
\end{tabular}

When executing MultiStart with 20 trial points, where results are presented in Table 7 , the improvements are more evident relatively to the ones obtained with the local solver.

Table 7. Optimization results with MultiStart for 20 trial points

\begin{tabular}{|c|c|c|c|c|c|}
\hline \multicolumn{2}{|c|}{ Output } & active-set & interior-point & SQP & trust-region- \\
\hline \multirow{2}{*}{\multicolumn{2}{|c|}{$\begin{array}{l}\text { Nr. function evaluations } \\
\text { Optimization time }[\mathrm{h}]\end{array}$}} & 3588 & 2887 & 3804 & 3200 \\
\hline & & $\sim 39.200$ & $\sim 31.500$ & $\sim 41.600$ & $\sim 35.000$ \\
\hline \multirow{3}{*}{$x$ global $[\mathrm{mm}]$} & $t$ & 9.990 & 10.135 & 9.990 & 9.990 \\
\hline & $w_{a}$ & 19.000 & 15.444 & 19.000 & 19.000 \\
\hline & $h_{b}$ & 10.000 & 14.513 & 10.000 & 10.000 \\
\hline \multicolumn{2}{|c|}{ Optimal global value $f(x)$} & 4.163 & 4.391 & 4.163 & 4.163 \\
\hline
\end{tabular}

The optimal value of function $f(x)$ improves $5.429 \%$ with active-set and trustregion-reflective algorithms compared with the value obtained with the local solver (4.402). The interior-point algorithm improves slightly $0.227 \%$ compared with the value obtained with the local solver (4.401). The optimal global value obtained with SQP algorithm remains in 4.163. The solver requires 3200 function evaluations and 35 hours to achieve the best optimal global value of the objective function with the trust-region-reflective algorithm, needing approximately 14 times more (1300\%) of optimization time and function evaluations than with the local solver. 


\subsection{Discussion of Results}

Following the discussion of results is presented for local and global solutions.

\section{Local Solution Discussion}

In general, the local solver fmincon converges to a good solution for the four algorithms, achieving a correlation of mode shapes and natural frequencies, between the reference and numerical model, with good quality.

The color graphs of Fig.4 represent the MAC matrix and frequencies matrix, and quantifies the correlation among the reference and numerical model. In $M A C$ matrix the diagonal should be as dark as possible and bright outside of the diagonal, to represent a good correlation among mode shapes, and the frequencies matrix should be as bright as possible to represent a good correlation among the frequencies.
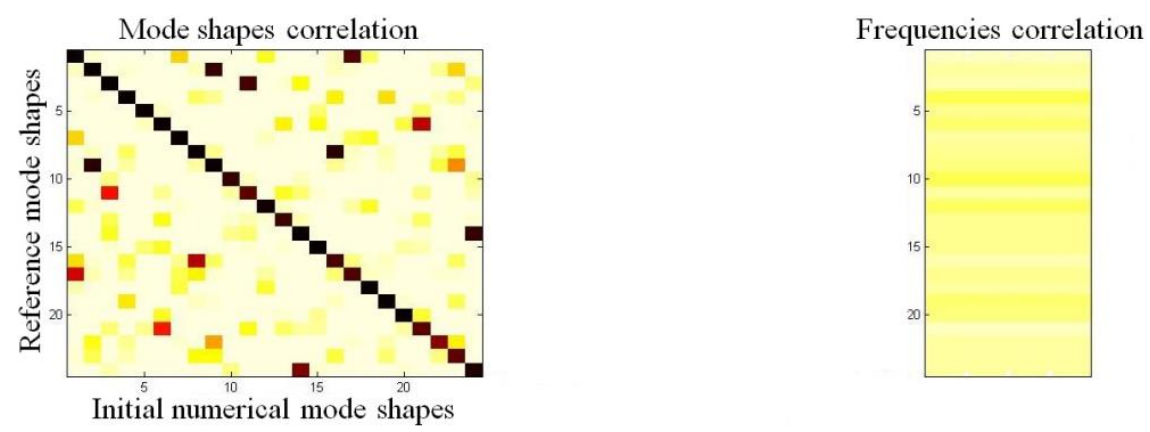

Fig. 4. Initial correlation

The value of first function evaluation, for any used algorithms, is 23.042 , because the initial point $x^{0}$ is the same for all cases. This value has the meaning of the geometric distance between the reference model and initial numerical model, imposed by initial variables of point $x^{0}$. This originates a weak correlation between, mainly, the natural frequency values of the two models, since the correlation between all mode shapes in diagonal MAC matrix is quite close to the unit, as shown in Fig.4.

After the optimization is complete, the quality of the natural frequencies correlation improves considerably, and reveals a slight improvement in MAC matrix, as shown in Fig.5a. The SQP algorithm is the one that achieves the best optimal value of objective function, and consequently, the best correlation among the two models.

The final numerical model, presented in Fig.5b, suffers significant changes due to the convergence of width $\left(w_{a}\right)$ and height $\left(h_{b}\right)$ parameters to the upper bounds. As the thickness $(t)$ parameter suffers a small change in relation to the initial value, the numerical model is now closer to the reference model, both geometrically and in terms of its dynamic behavior. 


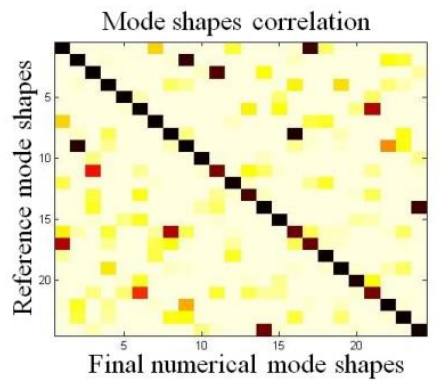

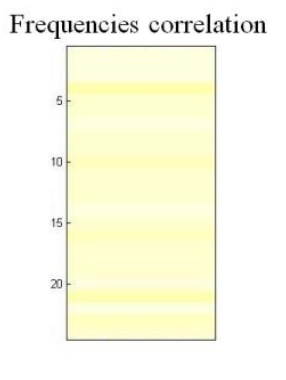

a)

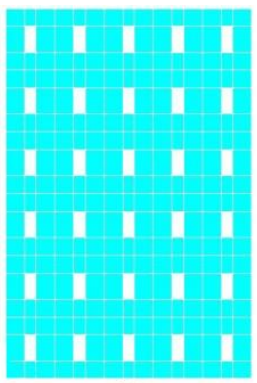

b)

Fig. 5. a) Best correlation for local optimization; b) Best final numerical model for local optimization

\section{Global Solution Discussion}

The global solvers, GlobalSearch and MultiStart are able to converge to a better solution than with the local solver fmincon, since we are facing a multimodal problem and they are prepared to find global solutions.

The MultiStart solver is the one that reveals more robustness in the set of the four algorithms. With 10 trial points, just the SQP algorithm is able to achieve the best optimal value of objective function (4.163), but with 20 trial points just interior-point algorithm does not achieves this value. The GlobalSearch solver does not reveal as robust as the MultiStart solver, because just the SQP algorithm is able to obtain the best optimal value of objective function $f(x)$. Despite the higher number of trial points, the GlobalSearch solver has the advantage of being able to select the best trail points among the starting points defined in the option NumStageOnePoints and reject the others. The solution obtained with the GlobalSearch solver using 100 trial points saves optimization time and function evaluations in approximately 3.5 times $(245.5 \%)$ face to MultiStart solver with 10 trial points and approximately 6.4 times $(536.4 \%)$ face to MultiStart solver with 20 trial points. Hence, the GlobalSearch solver is more efficient than Multistart since requires less computational effort to obtain the global solution.

The quality of the correlation between mode shapes and natural frequencies of the two models is presented in Fig.6a and illustrates the improvement relatively to the local solver when using a global solver.

The final numerical model, presented in Fig.6b, is closer to the reference model because the width $\left(w_{a}\right)$ parameter converges for the upper bound value. The height $\left(h_{b}\right)$ parameter keeps the initial value, and the thickness $(t)$ parameter suffers a small change, with regard to initial value, and converges to the lower bound. This parameter together with the other two, originates a final numerical model with very similar geometry and dynamic behavior in relation to the reference model. 


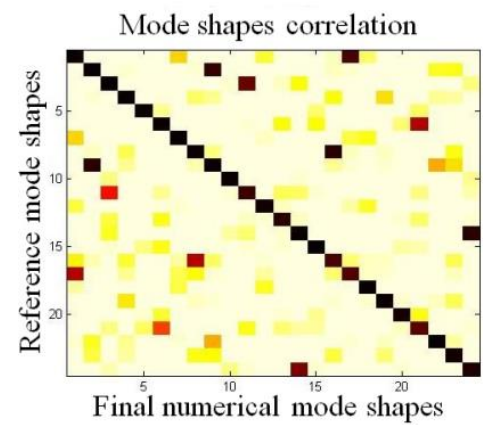

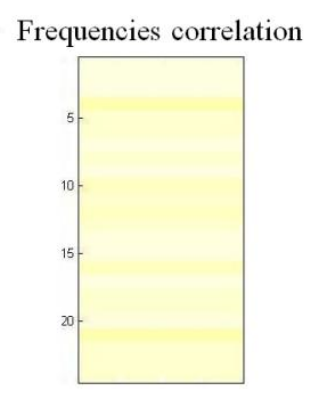

a)

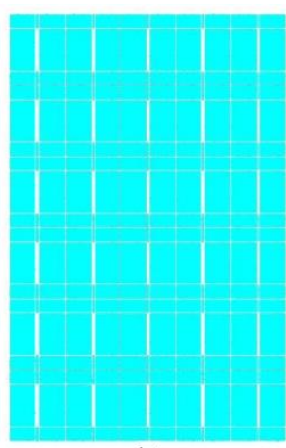

b)

Fig. 6. a) Best correlation for global optimization; b) Best final numerical model for global optimization

\section{Conclusions}

The aim of this paper was to apply two stochastic global optimization techniques for the optimization of a dynamic structural finite element model, and to establish a comparison with the previously local search method used in the Finite Element Model Updating program. The global solvers have the advantage of being able to work with a higher number of trial points, and therefore, are more efficient than the local solver. The two global solvers tested work in a different way, and therefore the results may also be different. Both global solvers achieve the same optimal global value of the objective function, requiring, however, different optimization times and function evaluations. In this case, the GlobalSearch is the fastest solver to achieve the best optimal global value when working with the SQP algorithm. The MultiStart solver achieved the same best optimal global value with the active-set, SQP and trustregion-reflective algorithms however needed six times more computational effort in terms of execution time and number of function evaluations.

The example used can be considered too oriented, which may increase the possibility of convergence of the local method, and somehow reduce the ability of perception of higher capacity of global methods. However, it was evident the evolution of the final numerical model to get closer to the geometry of the reference model when applied global optimization techniques.

In the future, more complex models will be studied and the use of stochastic global optimization methods based on Swarm Intelligence will be investigated.

Acknowledgments. The authors gratefully acknowledge the Centre for Mechanical and Materials Technologies (CT2M) and the Portuguese Funds through FCT "Fundação para a Ciência e a Tecnologia" under Project PEst-OE/EEI/UI0319/2014. 


\section{References}

1. Nocedal, J., Wright, S. J.: Numerical Optimization. Series in Operations Research, Springer Verlag, Heidelberg (1999)

2. Horst, R., Pardalos P.: Handbook of Global Optimization. Kluwer (1995)

3. Horst, R., Tuy, H.: Global Optimization Deterministic Approaches. Springer (1996)

4. Törn, A., Zilinskas, A.: Global Optimization. Springer-Verlag (1989)

5. Stolpe, M.: Global optimization of minimum weight truss topology problems with stress, displacement, and local buckling constraints using branch-and-bound. Int. J. Numer. Meth. Eng. 61, 1270-1309 (2004)

6. Achtziger, W., Stolpe M.: Truss topology optimization with discrete design variables Guaranteed global optimality and benchmark examples. Struct Multidisc Optim. 34, 1-20 (2007)

7. Lin, C.-Y., Chen W.-T.: Stochastic multistage algorithms for multimodal structural optimization. Comp. Struct. 24, 233-241 (2000)

8. Lucor, D., Enaux, C., Jourdren, H., Sagaut, P.: Stochastic design optimization: Application to reacting flows. Comput. Method Appl. M. 196, 5047-5062 (2007)

9. Birbil, S. I.: Stochastic Global Optimization Techniques, Faculty of North Carolina State University of Raleigh (2002)

10. Eriksson, P., Arora, J. S.: A comparison of global optimization algorithms applied to a ride comfort optimization problem. Struct. Multidisc. Optim. 24, 157-167 (2002)

11. Sonmez, F. O.: Shape optimization of $2 \mathrm{D}$ structures using simulated annealing. Comput. Method Appl. M. 196, 3279-3299 (2007)

12. Venanzi, I., Materazzi, A. L.: Multi-objective optimization of wind-excited structures. Eng. Struct. 23, 983-990 (2007)

13. Auvinen, P., Makela, M. M., Makinen, J.: Structural optimization of forest machines with hybridized nonsmooth and global methods. Struct. Multidisc. Optim. 23, 382-389 (2002)

14. Keller, D.: Global laminate optimization on geometrically partitioned shell structures. Struct Multidisc Optim., 43, 353-368 (2011)

15. Mottershead, J. E., Friswell, M. I.: Model updating in structural dynamics: A survey. J. Sound Vib. 167, 347-375 (1993)

16. Maia, N., Montalvão e Silva, J.: Theoretical and Experimental Modal Analysis. Hertfordshire: Research Studies Press Ltd (1997)

17. Levin, R., Lieven, N.: Dynamic Finite Element Model Updating Using Simulated Annealing and Genetic Algorithms, Mech. Syst. Signal Pr. 12, 91-120 (1998)

18. Teughels, A., De Roeck, G., Suykens, J. A. K.: Global Optimization by coupled local minimizers and its application to FE model updating. Comp. Struct. 81, 2337-2351 (2003)

19. Bakir, P. G., Reynders, E., De Roeck, G.: An improved finite element model updating method by the global optimization technique 'Coupled Local Minimizers'. Comp. Struct. 86, 1339-1352 (2008)

20. Ameri, E., Aghdam, M. M., Shakeri M.: Global optimization of laminated cylindrical panels based on fundamental natural frequency. Compos. Struct. 94, 2697-2705 (2012)

21. Meireles J.: Análise Dinâmica de Estruturas por Modelos de Elementos Finitos Identificados Experimentalmente, PhD Thesis, University of Minho (in Portuguese) (2007)

22. Allemang, R. J., Brown, D. L.: A Correlation Coefficient for Modal Vector Analysis. In Proceedings of the 1st International Modal Analysis Conference, Florida, Holiday Inn (1982)

23. MathWorks, Global Optimization Toolbox: User's Guide R2011b. Massachusetts: The MathWorksInc (2011) 
24. Voormeren, S., Rixen, R., Updating component reduction bases of static and vibration modes using preconditioned iterative techniques. Comput. Method Appl. M. 253, 39-59 (2013) 